\title{
Observation of a relatively low luminosity long duration GRB 201015A by the MAGIC telescopes
}

\author{
Yusuke Suda, ${ }^{a, *}$ Manuel Artero, ${ }^{b}$ Katsuaki Asano, ${ }^{c}$ Alessio Berti, ${ }^{d}$ Lara Nava, ${ }^{e, f}$ Koji \\ Noda $^{c}$ and Kenta Terauchi ${ }^{g}$ on behalf of the MAGIC Collaboration ${ }^{\dagger}$ \\ ${ }^{a}$ Physics Program, Graduate School of Advanced Science and Engineering, Hiroshima University, \\ 739-8526 Hiroshima, Japan \\ ${ }^{b}$ Institut de Física d'Altes Energies (IFAE), The Barcelona Institute of Science and Technology (BIST), \\ E-08193 Bellaterra (Barcelona), Spain \\ ${ }^{c}$ Institute for Cosmic Ray Research (ICRR), The University of Tokyo, Kashiwa, 277-8582 Chiba, Japan \\ ${ }^{d}$ Max-Planck-Institut für Physik, D-80805 München, Germany \\ ${ }^{e}$ National Institute for Astrophysics (INAF), I-00136 Rome, Italy \\ ${ }^{f}$ Istituto Nazionale Fisica Nucleare (INFN), I-34127 Trieste, Italy \\ ${ }^{g}$ Department of Physics, Kyoto University, 606-8502 Kyoto, Japan \\ E-mail: suda@astro.hiroshima-u.ac.jp
}

Starting from the first announcement of unequivocal detection of very high energy (VHE) emission from a gamma-ray burst (GRB) by the MAGIC telescopes (GRB 190114C), four additional detections of VHE emission from GRBs by ground-based telescopes were reported. These observations have revealed a new, energetic component that has become an additional probe to explore GRB physics. In order to deepen our understanding of the origin of this new component, and in general of the origin of radiation from GRBs, further observations by VHE instruments are crucial. In this work we report fast follow-up observations by the MAGIC telescopes of GRB 201015A, a GRB detected by the Swift/BAT. As measured by BAT, the prompt emission lasted $9.8 \pm 3.5$ seconds, suggesting that this GRB belongs to the class of long events. This was later confirmed by optical observations, which allowed to measure the redshift $(z=0.42)$ and found the associated type Ic-BL supernova. Having a prompt isotropic-equivalent energy of $E_{\text {iso }} \sim 10^{50} \mathrm{erg}$, this GRB is a relatively low energy event as compared to the population of long GRBs. Observations with the MAGIC telescopes started about 30 seconds after the GRB onset and were performed under good observational conditions. The accurate analysis of the MAGIC data reveals a strong hint of detection and implies a significant energy release in the $\mathrm{TeV}$ range, smaller but comparable with that of the prompt emission in the $\mathrm{keV}-\mathrm{MeV}$ band.

$37^{\text {th }}$ International Cosmic Ray Conference (ICRC 2021)

July 12 th $-23 r d, 2021$

Online - Berlin, Germany

\footnotetext{
${ }^{*}$ Presenter

${ }^{\dagger}$ a complete list of the MAGIC Collaboration authors can be found at the end of the proceedings
} 


\section{Introduction}

Gamma-ray bursts (GRBs) are the brightest explosions in the Universe. They are thought to be originated from the core collapse of massive stars or binary neutron star mergers. The duration of the initial brief emission (prompt emission) in the former events is longer than those in the latter events. A duration of 2 seconds is used to classify them as long or short GRBs. While the prompt emission lasts only several minutes at the longest and shows a complex light curve with sub-second variability, the subsequent emission (afterglow) is detected for several days or longer and its flux, after an initial rise smoothly decreases as a power-law (PL) in time. Once space-borne telescopes detect the prompt emission from GRBs in X-ray and gamma-ray bands, several instruments initiate follow-up observations after receiving the alert from satellites, providing multi-wavelength observations of the afterglow phase.

Afterglow radiation is thought to be produced when the ultra-relativistic jet collides with the surrounding medium. In most cases, multi-wavelength afterglow observations from radio to $\mathrm{GeV}$ gamma-rays can be explained by electron synchrotron radiation from the forward shock, with a possible early time contribution from the reverse shock running into the jet. The ground-breaking discovery of a new component beyond synchrotron was made by the MAGIC telescopes with the detection of TeV gamma-rays from the afterglow of the long GRB 190114C [1]. This burst was very energetic $\left(E_{\text {iso }} \sim 3 \times 10^{53} \mathrm{erg}\right.$ ) and also one of the closest GRBs ever followed by MAGIC. The observational delay of the MAGIC telescopes was one of the shortest, being around 60 seconds. Together with other multi-wavelength data, the TeV emission from GRB 190114C is consistent with being synchrotron self-Compton radiation, and is found to carry a power comparable to that of synchrotron component [2]. Moreover, the afterglow parameters inferred from the multi-wavelength modeling are quite typical for GRBs, suggesting that $\mathrm{TeV}$ emission might be commonly in GRB afterglows, at least for bright GRBs.

After GRB 190114C, a few other detections of VHE gamma-ray emission in the TeV range from long GRBs have been reported, both by H.E.S.S. (GRB 180720B [3] and GRB 190829A [4]) and MAGIC (GRB 201216C [5]). In order to understand and confirm how TeV emission is produced and whether there might be more than one production mechanism efficiently producing VHE emission, a larger sample of TeV GRBs is needed. In this proceeding, we report a strong hint of detection of VHE gamma-ray emission from a relatively low luminosity GRB 201015A observed by the MAGIC telescopes.

\section{GRB 201015A}

GRB 201015A has been detected by the Swift-BAT on 15 October 2020, 22:50:13 universal time (UT) (hereafter $T_{0}$ ) [6]. The prompt duration $T_{90}$, estimated as the duration of the time interval during which between $5 \%$ and $95 \%$ of the total photon counts are detected, is $T_{90}=(9.78 \pm 3.47) \mathrm{s}$ in the $15-350 \mathrm{keV}$ band. The BAT spectrum is well modeled by a single PL with photon index $\beta=-3.03 \pm 0.68$, suggesting a low peak energy $E_{\mathrm{p}}<10 \mathrm{keV}$. The fluence in the $15-150 \mathrm{keV}$ band is $S_{\gamma \text {,iso }}=(2.0 \pm 0.6) \times 10^{-7} \mathrm{erg} / \mathrm{cm}^{2}$ [7]. There was no onboard trigger by the Fermi-GBM around $T_{0}$, but a transient source, whose location is consistent with the Swift-BAT event, was identified by the GBM targeted search [8]. 
The GTC telescope reported a measurement of the redshift $z=0.426$ [9], later confirmed by the NOT $(z=0.423[10])$. The (isotropic equivalent) prompt emission energy inferred from spectral analysis of Fermi-GBM data is $E_{\gamma \text {,iso }}=(1.1 \pm 0.2) \times 10^{50} \mathrm{erg}$ [11].

Due to an observing constraint, Swift-XRT started observations $3214 \mathrm{~s}$ after $T_{0}$. Observations up to almost 1 day show that the XRT flux decays as a PL with index $\alpha \sim 1.49$ [14]. Observations by the Chandra X-ray Observatory at later times (8.4 days and 13.6 days after the burst) are inconsistent with the extrapolation of the PL decay identified by XRT, having a flux about 100 times higher than expected [16]. Late time observations (between 18 and 21 days) by XRT confirmed a flattening in the X-ray lightcurve [15].

Inside the Swift-BAT error-box, an optical transient was found by the MASTER-Tavrida robotic telescope with a $30 \mathrm{~s}$ exposure taken $168 \mathrm{~s}$ after $T_{0}$ [12], which was later confirmed by the other optical telescopes. Preliminary optical magnitudes for the filters $g, r, i$ reported by the NUTTelATAO show a clear initial rise, a peak around $200 \mathrm{~s}$, followed by a decay [13]. The VLA detected a bright radio source with a flux density of about $1.3 \times 10^{-4} \mathrm{Jy}$ at $6 \mathrm{GHz} 1.41$ days after the burst, whose location is consistent with the optical afterglow position [17]. Late time optical observations identified an associated supernova [18]. The supernova component rose 5 days after the burst and its maximum is between 12 days and 20 days after $T_{0}$. The supernova evidence was also confirmed by the LBT telescope [19].

\section{MAGIC observations}

The MAGIC telescopes consist of two $17 \mathrm{~m}$ diameter imaging atmospheric Cherenkov telescopes, located at the Roque de Los Muchachos Observatory in La Palma, Canary Islands, Spain. The telescopes can detect VHE gamma-rays above $50 \mathrm{GeV}$, with an integral sensitivity above $220 \mathrm{GeV}$ corresponding to about $0.66 \%$ of the Crab Nebula flux in $50 \mathrm{~h}$ of observations [20]. Thanks to their light-weight structure, the telescopes can point to a target with a repositioning speed of $7^{\circ} \mathrm{s}^{-1}$.

On 15 October 2020, at 22:50:32 UT $\left(T_{0}+19 \mathrm{~s}\right)$, the automatic alert system received an alert by the Swift-BAT with the preliminary coordinates of GRB 201015A (right ascension, $23 \mathrm{~h} 37 \mathrm{~m}$ $22 \mathrm{~s}$; declination, $+53 \mathrm{~d} 23 \mathrm{~m} 36 \mathrm{~s}$ ). The alert was validated as observable by the system and the automatic repointing procedure started immediately. The MAGIC telescopes started tracking on the target, and observations began at $T_{0}+33 \mathrm{~s}$. The observations were performed under dark conditions and lasted $4 \mathrm{~h}$ after $T_{0}$. The initial zenith angle of the telescopes was $24^{\circ}$ and reached $48^{\circ}$ at the end of observations. Quick offline analyses showed a hint of gamma-ray signal from the location of the GRB with significance $>3 \sigma$, that was reported in the Gamma-ray Coordinates Network [21].

Weather conditions during the observation were good for most of the time. However, a total $0.45 \mathrm{~h}$ of data in the last half of the observations were estimated to be affected by passing clouds in the field of view of the telescopes. After removing these affected data, we performed the data analysis with the standard MAGIC analysis software [22]. The energy threshold is evaluated to be $140 \mathrm{GeV}$ from Monte Carlo simulated gamma-ray data. 


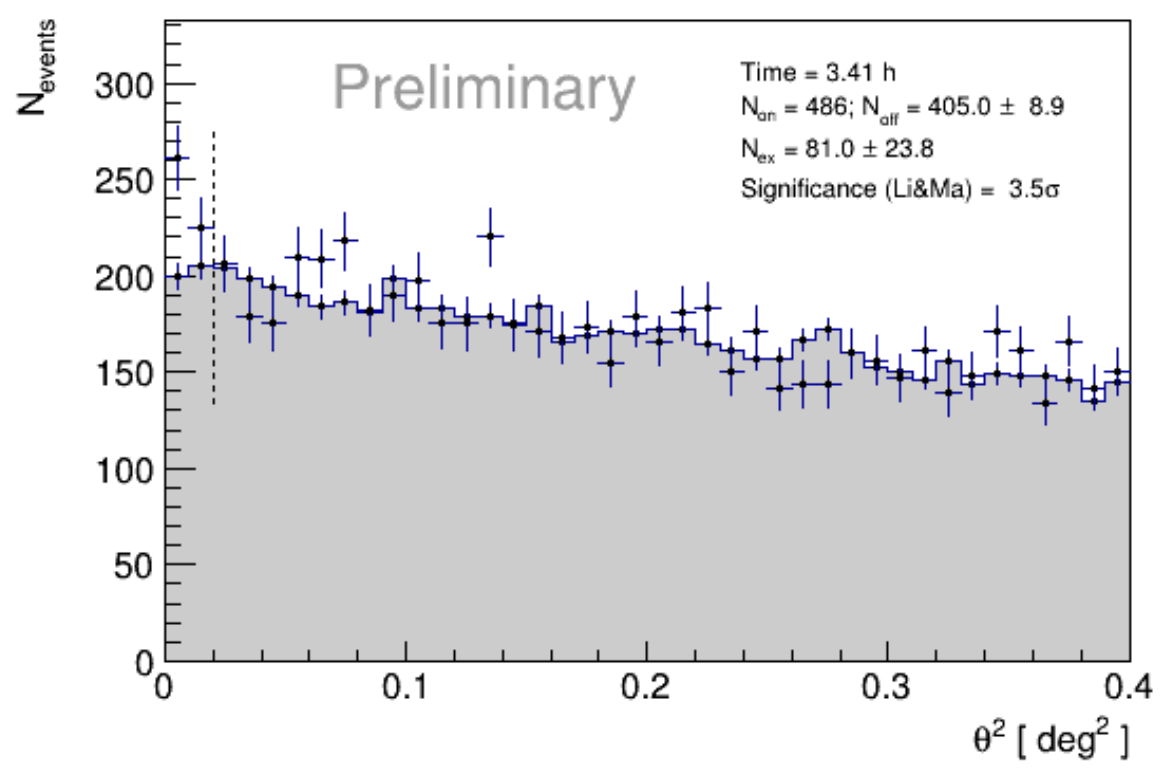

Figure 1: Distribution of the squared angular distance $\left(\theta^{2}\right)$ between the position of GRB 201015A and the reconstructed position of the events.

\section{Results}

The distribution of the squared angular distance $\left(\theta^{2}\right)$ between the target position and the

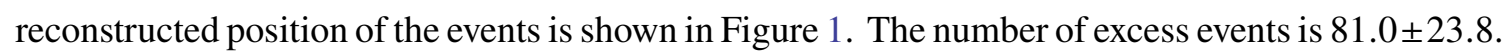
The significance of the gamma-ray signal at the GRB position is calculated using Equation (17) of Li\&Ma [23], and is $3.5 \sigma$. Figure 2 shows a significance (test statistic) sky map, centered at the GRB refined coordinates by the NOT (right ascension, $23 \mathrm{~h} 37 \mathrm{~m} 16.41 \mathrm{~s}$; declination, $+53 \mathrm{~d} 24 \mathrm{~m}$ $56.5 \mathrm{~s}$ [24]). At this position, we find a significance compatible with the one reported in Figure 1.

\section{Discussion and conclusion}

We presented the analysis of MAGIC observations performed starting $~ 33$ seconds after the onset of GRB 201015A. A strong hint of detection (at the level of $3.5 \sigma$ ) is found. The energy released in the VHE range is smaller but comparable to the one released during the prompt emission phase. A further refinement of the data analysis is planned, to confirm the significance of the excess. GRB 201015A would be one of the five GRBs detected by a Cherenkov telescope, together with GRB 190114C, GRB 180720B, GRB 190829A, and GRB 201216C. Also in this case, the GRB belongs to the class of long events, and is detected at $\mathrm{TeV}$ energies during its afterglow phase. Located at a distance very similar to that of GRB 190114C $(z \sim 0.42)$, its energy release in the prompt phase was however about $3 \times 10^{3}$ times smaller $\left(E_{\gamma \text {,iso }} \sim 10^{50} \mathrm{erg}\right)$. In spite of this, the MAGIC telescopes found evidence of $>140 \mathrm{GeV}$ emission from this relatively faint GRB. From preliminary estimates, we find that the ratio between the $\mathrm{TeV}$ flux at a given time and the prompt emission energy is the same for the two GRBs. 


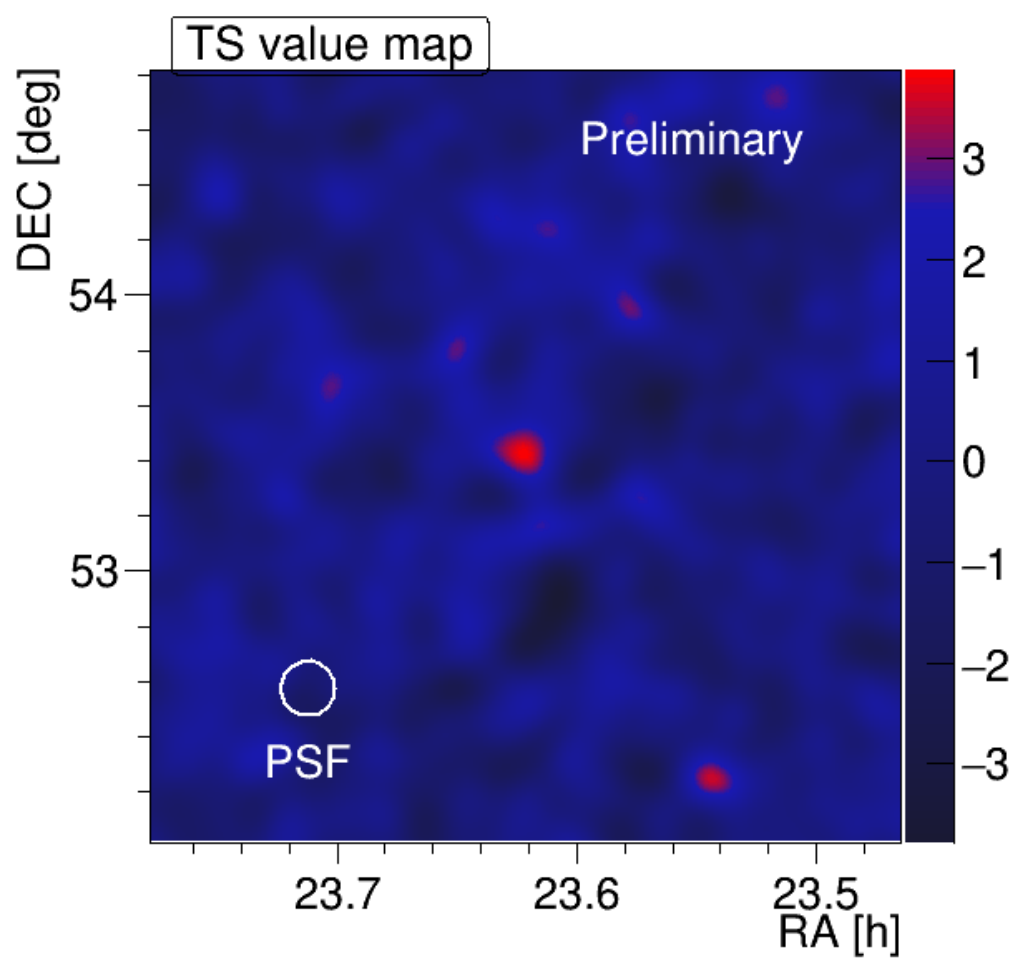

Figure 2: Significance sky map.

The possibility that GRBs with a relatively low energetic can produce $\mathrm{TeV}$ radiation at a level that is detectable also from relatively large $(z=0.4)$ redshift has of course a large impact both on theoretical models and on future prospects for detections of GRBs with the current and the upcoming generation of Cherenkov telescopes.

\section{Acknowledgements}

We acknowledge the support from the agencies and organizations listed here: https:// magic.mpp.mpg . de/acknowledgments_ICRC2021

\section{References}

[1] MAGIC Collaboration. Teraelectronvolt emission from the $\gamma$-ray burst GRB 190114C. Nature, $575,455-458$ (2019)

[2] MAGIC Collaboration et al. Observation of inverse Compton emission from a long $\gamma$-ray burst. Nature, 575, 459-463 (2019)

[3] H. Abdalla et al. A very-high-energy component deep in the $\gamma$-ray burst afterglow. Nature, 575, 464-467 (2019)

[4] H.E.S.S. Collaboration et al. Revealing x-ray and gamma ray temporal and spectral similarities in the GRB 190829A afterglow. Science, 372, 1081-1085 (2021) 
[5] S. Fukami et al. Very-high-energy gamma-ray emission from GRB 201216C detected by MAGIC. in these proceedings (2021)

[6] V. D'Elia et al. GRB 201015A: Swift detection of a burst. GCN Circulars 28632 (2020)

[7] C. B. Markwardt et al. GRB 201015A: Swift-BAT refined analysis (a soft short pulse with a tail emission). GCN Circulars 28658 (2020)

[8] C. Fletcher et al. Fermi GBM Sub-Threshold Detection of GRB 201015A. GCN Circulars $28663(2020)$

[9] A. de Ugarte Postigo et al. GRB 201015A: Redshift from GTC/OSIRIS. GCN Circulars 28649 (2020)

[10] L. Izzo et al. GRB 201015A: redshift confirmation. GCN Circulars 28661 (2020)

[11] P. Minaev and A. Pozanenko on behalf of larger GRB follow-up collaboration. GRB 201015A: classification as long GRB. GCN Circulars 28668 (2020)

[12] V. Lipunov et al. Swift GRB201015.95: Global MASTER-Net OT detection. GCN Circulars $28633(2020)$

[13] B. Grossan et al. GRB 201015A NUTTelA-TAO / BSTI Early Measurements (Preliminary). GCN Circulars 28674 (2020)

[14] A. D'Ai et al. GRB 201015A: Swift-XRT refined Analysis. GCN Circulars 28660 (2020)

[15] V. D'Elia on behalf of the Swift team. GRB 201015A: Swift-XRT late time observations. GCN Circulars 28857 (2020)

[16] B. Gompertz et al. GRB 201015A: Late X-ray Detections with Chandra. GCN Circulars 28822 (2020)

[17] W. Fong et al. GRB 201015A: $6 \mathrm{GHz}$ VLA radio afterglow candidate detection. GCN Circulars $28688(2020)$

[18] A. Pozanenko et al. GRB 201015A: optical observations and supernova identification. GCN Circulars 29033 (2020)

[19] A. Rossi et al. GRB 201015A: evidence of supernova in LBT spectra. GCN Circulars 29306 (2020)

[20] J. Aleksic et al. The major upgrade of the MAGIC telescopes, Part II: A performance study using observations of the Crab Nebula. APh, 72, 76 (2016)

[21] O. Blanch et al. MAGIC observations of GRB 201015A: hint of very high energy gamma-ray signal. GCN Circulars 28659 (2020)

[22] R. Zanin et al. MARS, The MAGIC Analysis and Reconstruction Software. ICRC (Rio de Janeiro) (2013) 
[23] T. P. Li and Y. Q. Ma. Analysis methods for results in gamma-ray astronomy. ApJ, 272, 317 (1983)

[24] D. B. Malesani et al. GRB 201015A: NOT optical afterglow confirmation. GCN Circulars $28637(2020)$

\section{The MAGIC Collaboration}

V. A. Acciari ${ }^{1}$, S. Ansoldi ${ }^{2,41}$, L. A. Antonelli ${ }^{3}$, A. Arbet Engels $^{4}$, M. Artero ${ }^{5}$, K. Asano $^{6}$, D. Baack ${ }^{7}$, A. Babié ${ }^{8}$, A. Baquero ${ }^{9}$, U. Barres de Almeida ${ }^{10}$, J. A. Barrio ${ }^{9}$, I. Batković ${ }^{11}$, J. Becerra González ${ }^{1}$, W. Bednarek ${ }^{12}$, L. Bellizzi ${ }^{13}$, E. Bernardini ${ }^{14}$, M. Bernardos ${ }^{11}$, A. Berti ${ }^{15}$, J. Besenrieder ${ }^{15}$, W. Bhattacharyya ${ }^{14}$, C. Bigongiari ${ }^{3}$, A. Biland ${ }^{4}$, O. Blanch ${ }^{5}$, H. Bökenkamp ${ }^{7}$, G. Bonnoli ${ }^{16}$, Ž. Bošnjak $^{8}$, G. Busetto ${ }^{11}$, R. Carosi ${ }^{17}$, G. Ceribella ${ }^{15}$, M. Cerruti ${ }^{18}$, Y. Chai ${ }^{15}$, A. Chilingarian ${ }^{19}$, S. Cikota ${ }^{8}$, S. M. Colak ${ }^{5}$, E. Colombo ${ }^{1}$, J. L. Contreras ${ }^{9}$, J. Cortina ${ }^{20}$, S. Covino ${ }^{3}$, G. D’Amico ${ }^{15,42}$, V. D’Elia ${ }^{3}$, P. Da Vela ${ }^{17,43}$, F. Dazzi ${ }^{3}$, A. De Angelis ${ }^{11}$, B. De Lotto ${ }^{2}$, M. Delfino ${ }^{5,44}$, J. Delgado ${ }^{5,44}$, C. Delgado Mendez ${ }^{20}$, D. Depaoli ${ }^{21}$, F. Di Pierro ${ }^{21}$, L. Di Venere ${ }^{22}$, E. Do Souto Espiñeira ${ }^{5}$, D. Dominis Prester ${ }^{23}$, A. Donini ${ }^{2}$, D. Dorner ${ }^{24}$, M. Doro ${ }^{11}$, D. Elsaesser ${ }^{7}$, V. Fallah Ramazani ${ }^{25,45}$, A. Fattorini ${ }^{7}$, M. V. Fonseca ${ }^{9}$, L. Font ${ }^{26}$, C. Fruck ${ }^{15}$, S. Fukami ${ }^{6}$, Y. Fukazawa ${ }^{27}$, R. J. García López ${ }^{1}$, M. Garczarczyk ${ }^{14}$, S. Gasparyan ${ }^{28}$, M. Gaug ${ }^{26}$, N. Giglietto $^{22}$, F. Giordano ${ }^{22}$, P. Gliwny ${ }^{12}$, N. Godinović ${ }^{29}$, J. G. Green ${ }^{3}$, D. Green ${ }^{15}$, D. Hadasch ${ }^{6}$, A. Hahn ${ }^{15}$, L. Heckmann ${ }^{15}$, J. Herrera ${ }^{1}$, J. Hoang 9,46 , D. Hrupec ${ }^{30}$, M. Hütten ${ }^{15}$, T. Inada ${ }^{6}$, K. Ishio ${ }^{12}$, Y. Iwamura ${ }^{6}$, I. Jiménez Martínez ${ }^{20}$, J. Jormanainen ${ }^{25}$, L. Jouvin ${ }^{5}$, M. Karjalainen ${ }^{1}$, D. Kerszberg ${ }^{5}$, Y. Kobayashi ${ }^{6}$, H. Kubo ${ }^{31}$, J. Kushida ${ }^{32}$, A. Lamastra ${ }^{3}$, D. Lelas ${ }^{29}$, F. Leone ${ }^{3}$, E. Lindfors ${ }^{25}$, L. Linhoff ${ }^{7}$, S. Lombardi ${ }^{3}$, F. Longo ${ }^{2,47}$, R. López-Coto ${ }^{11}$, M. López-Moya ${ }^{9}$, A. López-Oramas ${ }^{1}$, S. Loporchio ${ }^{22}$, B. Machado de Oliveira Fraga ${ }^{10}$, C. Maggio ${ }^{26}$, P. Majumdar ${ }^{33}$, M. Makariev ${ }^{34}$, M. Mallamaci ${ }^{11}$, G. Maneva ${ }^{34}$, M. Manganaro ${ }^{23}$, K. Mannheim² ${ }^{24}$, L. Maraschi ${ }^{3}$, M. Mariotti ${ }^{11}$, M. Martínez ${ }^{5}$, D. Mazin ${ }^{6,15}$, S. Menchiari ${ }^{13}$, S. Mender ${ }^{7}$, S. Mićanović2 ${ }^{23}$, D. Miceli ${ }^{2,49}$, T. Miener ${ }^{9}$, J. M. Miranda ${ }^{13}$, R. Mirzoyan ${ }^{15}$, E. Molina ${ }^{18}$, A. Moralejo ${ }^{5}$, D. Morcuende ${ }^{9}$, V. Moreno ${ }^{26}$, E. Moretti ${ }^{5}$, T. Nakamori ${ }^{35}$, L. Nava ${ }^{3}$,

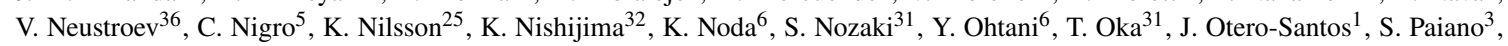
M. Palatiello ${ }^{2}$, D. Paneque ${ }^{15}$, R. Paoletti ${ }^{13}$, J. M. Paredes ${ }^{18}$, L. Pavletić ${ }^{23}$, P. Peñil ${ }^{9}$, M. Persic ${ }^{2,50}$, M. Pihet ${ }^{15}$, P. G. Prada Moroni ${ }^{17}$, E. Prandini ${ }^{11}$, C. Priyadarshi ${ }^{5}$, I. Puljak ${ }^{29}$, W. Rhode ${ }^{7}$, M. Ribó ${ }^{18}$, J. Rico ${ }^{5}$, C. Righi ${ }^{3}$, A. Rugliancich ${ }^{17}$, N. Sahakyan ${ }^{28}$, T. Saito ${ }^{6}$, S. Sakurai ${ }^{6}$, K. Satalecka ${ }^{14}$, F. G. Saturni ${ }^{3}$, B. Schleicher ${ }^{24}$, K. Schmidt ${ }^{7}$, T. Schweizer ${ }^{15}$, J. Sitarek ${ }^{12}$, I. Šnidaric ${ }^{37}$, D. Sobczynska ${ }^{12}$, A. Spolon ${ }^{11}$, A. Stamerra ${ }^{3}$, J. Strišković ${ }^{30}$, D. Strom ${ }^{15}$, M. Strzys ${ }^{6}$, Y. Suda ${ }^{27}$, T. Surić ${ }^{37}$, M. Takahashi ${ }^{6}$, R. Takeishi ${ }^{6}$, F. Tavecchio ${ }^{3}$, P. Temnikov ${ }^{34}$, T. Terzić ${ }^{23}$, M. Teshima ${ }^{15,6}$, L. Tosti $^{38}$, S. Truzzi $^{13}$, A. Tutone ${ }^{3}$, S. Ubach ${ }^{26}$, J. van Scherpenberg ${ }^{15}$, G. Vanzo $^{1}$, M. Vazquez Acosta ${ }^{1}$, S. Ventura ${ }^{13}$, V. Verguilos ${ }^{34}$, C. F. Vigorito ${ }^{21}$, V. Vitale ${ }^{39}$, I. Vovk ${ }^{6}$, M. Will ${ }^{15}$, C. Wunderlich ${ }^{13}$, T. Yamamoto $^{40}$, and D. Zarić ${ }^{29}$

${ }^{1}$ Instituto de Astrofísica de Canarias and Dpto. de Astrofísica, Universidad de La Laguna, E-38200, La Laguna, Tenerife, Spain ${ }^{2}$ Università di Udine and INFN Trieste, I-33100 Udine, Italy ${ }^{3}$ National Institute for Astrophysics (INAF), I-00136 Rome, Italy ${ }^{4}$ ETH Zürich, CH-8093 Zürich, Switzerland ${ }^{5}$ Institut de Física d'Altes Energies (IFAE), The Barcelona Institute of Science and Technology (BIST), E-08193 Bellaterra (Barcelona), Spain ${ }^{6}$ Japanese MAGIC Group: Institute for Cosmic Ray Research (ICRR), The University of Tokyo, Kashiwa, 277-8582 Chiba, Japan ${ }^{7}$ Technische Universität Dortmund, D-44221 Dortmund, Germany ${ }^{8}$ Croatian MAGIC Group: University of Zagreb, Faculty of Electrical Engineering and Computing (FER), 10000 Zagreb, Croatia ${ }^{9}$ IPARCOS Institute and EMFTEL Department, Universidad Complutense de Madrid, E-28040 Madrid, Spain ${ }^{10}$ Centro Brasileiro de Pesquisas Físicas (CBPF), 22290-180 URCA, Rio de Janeiro (RJ), Brazil ${ }^{11}$ Università di Padova and INFN, I-35131 Padova, Italy ${ }^{12}$ University of Lodz, Faculty of Physics and Applied Informatics, Department of Astrophysics, 90-236 Lodz, Poland ${ }^{13}$ Università di Siena and INFN Pisa, I-53100 Siena, Italy ${ }^{14}$ Deutsches Elektronen-Synchrotron (DESY), D-15738 Zeuthen, Germany ${ }^{15}$ Max-Planck-Institut für Physik, D-80805 München, Germany ${ }^{16}$ Instituto de Astrofísica de Andalucía-CSIC, Glorieta de la Astronomía s/n, 18008, Granada, Spain ${ }^{17}$ Università di Pisa and INFN Pisa, I-56126 Pisa, Italy ${ }^{18}$ Universitat de Barcelona, ICCUB, IEEC-UB, E-08028 Barcelona, Spain ${ }^{19}$ Armenian MAGIC Group: A. Alikhanyan National Science Laboratory, 0036 Yerevan, Armenia ${ }^{20}$ Centro de Investigaciones Energéticas, Medioambientales y Tecnológicas, E-28040 Madrid, Spain ${ }^{21}$ INFN MAGIC Group: INFN Sezione di Torino and Università degli Studi di Torino, I-10125 Torino, Italy ${ }^{22}$ INFN MAGIC Group: INFN Sezione di Bari and Dipartimento Interateneo di Fisica dell'Università e del Politecnico di Bari, I-70125 Bari, Italy ${ }^{23}$ Croatian MAGIC Group: University of Rijeka, Department of Physics, 51000 Rijeka, Croatia ${ }^{24}$ Universität Würzburg, D-97074 Würzburg, Germany ${ }^{25}$ Finnish MAGIC Group: Finnish Centre for Astronomy with ESO, University of Turku, FI-20014 Turku, Finland ${ }^{26}$ Departament de Física, and CERES-IEEC, Universitat Autònoma de Barcelona, E-08193 Bellaterra, Spain ${ }^{27}$ Japanese MAGIC Group: Physics Program, Graduate School of Advanced Science and Engineering, Hiroshima University, 739-8526 Hiroshima, Japan ${ }^{28}$ Armenian MAGIC Group: ICRANet-Armenia at NAS RA, 0019 Yerevan, Armenia ${ }^{29}$ Croatian MAGIC Group: University of Split, Faculty of Electrical Engineering, Mechanical Engineering and Naval Architecture (FESB), 21000 Split, Croatia ${ }^{30}$ Croatian MAGIC Group: Josip Juraj Strossmayer University of Osijek, Department of Physics, 31000 Osijek, Croatia ${ }^{31}$ Japanese MAGIC Group: Department of Physics, Kyoto University, 606-8502 Kyoto, Japan ${ }^{32}$ Japanese MAGIC Group: Department of Physics, Tokai University, Hiratsuka, 259-1292 Kanagawa, Japan ${ }^{33}$ Saha Institute of Nuclear Physics, HBNI, 1/AF Bidhannagar, Salt Lake, Sector-1, Kolkata 700064, India ${ }^{34}$ Inst. for Nucl. Research and Nucl. Energy, Bulgarian Academy of Sciences, BG-1784 Sofia, Bulgaria ${ }^{35}$ Japanese MAGIC Group: Department of Physics, Yamagata University, Yamagata 990-8560, Japan ${ }^{36}$ Finnish MAGIC 
Group: Astronomy Research Unit, University of Oulu, FI-90014 Oulu, Finland ${ }^{37}$ Croatian MAGIC Group: Ruđer Bošković Institute, 10000 Zagreb, Croatia ${ }^{38}$ INFN MAGIC Group: INFN Sezione di Perugia, I-06123 Perugia, Italy ${ }^{39}$ INFN MAGIC Group: INFN Roma Tor Vergata, I-00133 Roma, Italy ${ }^{40}$ Japanese MAGIC Group: Department of Physics, Konan University, Kobe, Hyogo 6588501, Japan ${ }^{41}$ also at International Center for Relativistic Astrophysics (ICRA), Rome, Italy ${ }^{42}$ now at Department for Physics and Technology, University of Bergen, NO-5020, Norway ${ }^{43}$ now at University of Innsbruck ${ }^{44}$ also at Port d'Informació Científica (PIC), E-08193 Bellaterra (Barcelona), Spain ${ }^{45}$ now at Ruhr-Universität Bochum, Fakultät für Physik und Astronomie, Astronomisches Institut (AIRUB), 44801 Bochum, Germany ${ }^{46}$ now at Department of Astronomy, University of California Berkeley, Berkeley CA $94720{ }^{47}$ also at Dipartimento di Fisica, Università di Trieste, I-34127 Trieste, Italy ${ }^{49}$ now at Laboratoire d'Annecy de Physique des Particules (LAPP), CNRS-IN2P3, 74941 Annecy Cedex, France ${ }^{50}$ also at INAF Trieste and Dept. of Physics and Astronomy, University of Bologna, Bologna, Italy 